\title{
SALUD PERCIBIDA POR LAS PERSONAS MAYORES EN ESPAÑA EN FUNCIÓN DEL TIPO DE DEMANDA DE ACTIVIDAD FÍSICA
}

\author{
HEALTH PERCEIVED BY OLDER PEOPLE IN SPAIN REGARDING THE \\ TYPE OF PHYSICAL ACTIVITY DEMAND
}

\section{SAÚDE PERCEBIDA PELAS PESSOAS MAIS VELHAS NA ESPANHA DE ACORDO COM O TIPO DE DEMANDA PARA A ATIVIDADE FÍSICA}

\author{
María Espada Mateos*, José Carlos Calero Cano*
}

\section{Palabras clave:}

Salud.

Anciano.

Ejercicio.

Análisis

demográfico.
Keywords:

Health.

Elderly.

Exercise.

Demographic

analysis.

Palavras chave:

Saúde.

Idoso.

Exercício.

Análise demográfica.
Resumen: Actualmente asistimos a un lento e inevitable proceso de envejecimiento de la población. Este hecho convierte a las personas mayores en un grupo poblacional de excepcional interés para las investigaciones científicas. Uno de los objetivos mas presentes en la literatura es el de mejorar su salud y su calidad de vida, logrando un envejecimiento activo. Por ello, el objetivo principal de la presente investigación es conocer cómo perciben las personas mayores su salud en función del grupo de demanda de actividad física en el que se encuentran, estableciéndose, además, relaciones con las variables sociodemográficas. La metodología ha consistido en una encuesta seccional aplicada a una muestra estadísticamente representativa de personas mayores de 64 años en España. Los resultados muestran que las personas físicamente activas perciben mejor su estado de salud y que las diferencias sociodemográficas se reducen o invierten en este grupo.

Abstract: We witness a slow and inevitable process of demographic aging of the population, which makes the elderly a population group of particular interest in our field of research. Literature focuses on improving their health and quality of life through active aging. For that reason, the main goal of this research was to learn how the elderly perceive their health regarding the different types of demand for physical activity, relating them to socio-demographic variables. The methodology consisted in a sectional survey applied to a statistically representative sample of over-64 seniors in Spain. The results suggest that physically active people have better perception of their health. Furthermore, socio-demographic differences were reduced or reverted in that group.

Resumo: Assistimos atualmente a um processo lento e inevitável do envelhecimento da população no mundo. Esse fato converte as pessoas mais velhas em um grupo populacional de excepcional interesse para as investigações científicas. Um dos objetivos mais discutidos na literatura científica é procurar melhorar a saúde e a qualidade de vida, para assim obter um envelhecimento ativo. Então, o objetivo principal desta pesquisa é conhecer como as pessoas mais velhas percebem sua saúde de acordo com a demanda do grupo da atividade física que elas participam, além de estabelecer relações com as variáveis sócio-demográficas. A metodologia constituiu-se em um estudo seccional aplicado a uma amostra estatisticamente representativa de pessoas com mais de 64 anos na Espanha. Os resultados mostraram que as pessoas fisicamente ativas percebem melhor seu estado de saúde e as diferenças sócio-demográficas são reduzidas ou revertidas neste grupo.
*Universidad Pontificia de Comillas. Universidad Camilo José Cela. Universidad Internacional de La Rioja. Madrid, España. E-mail: mariaespada_mateos@ hotmail.com

${ }^{*}$ Centro de Estudios Guzmán el Bueno. Madrid, España.

E-mail: jc.calero@ hotmail.es

Recebido em: 13-11-2014

Aprovado em: 15-06-2015

(c) (1) () Licence 


\section{INTRODUCCIÓN}

Cuando se hace referencia a las personas mayores, se atiende a una fuerza demográfica y a un grupo social en continua expansión, que difícilmente puede cambiar en los próximos años, debido a la bajísima natalidad y mortalidad y el constante aumento de la esperanza de vida, es lo que los expertos denominan "revolución silenciosa", que tiende a invertir la pirámide de la población (GARCÍA, 2005). Este crecimiento es más acusado en las sociedades occidentales (CAMPOS et al., 2011).

En España, a fecha de 1 de noviembre de 2011, el 17,33\% del total de la población son personas mayores de 64 años (I.N.E, 2011-2012), constatándose además que la edad media desde el año 2001 aumentó en 1,5 años. Por otra parte, los resultados del la Encuesta Nacional de Salud realizada en 2011/2012 por el Instituto Nacional de Estadística (I.N.E.) revelaron un aumento de las patologías crónicas, de forma que la hipertensión arterial, el colesterol elevado, la obesidad y la diabetes siguen su tendencia ascendente, especialmente entre la población de más edad.

En este sentido, Romero (2002) señala que el $41 \%$ de las personas mayores en España se encuentran afectada por algún tipo de enfermedad crónica de tipo cardiocirculatoria, hipertensión, falta de riego etc. y que el 35\% de las personas muy mayores presentan lesiones osteoarticulares, con especial incidencia en las de tipo degenerativo. Asimismo, este autor expone que un $14 \%$ sufre problemas de tipo respiratorio, digestivo o nervioso, y un $10 \%$ padece algún tipo de alteración crónica metabólica.

Por otra parte, en el estudio de Peerson y While (2011) realizado en Londres, el 46,6\% de las personas mayores presentan hipertensión arterial, siendo éste el principal problema de salud encontrado, aunque también registraron problemas de cáncer, artritis, fracturas, diabetes y obesidad. Cabe destacar que para dichos autores algunos de estos problemas se asocian al bajo nivel de práctica deportiva.

Atendiendo a la calidad de vida de las personas en relación a su salud, no se deben tener únicamente en cuenta los aspectos físicos o biológicos, sino que se deben tener en cuenta todos los planos de la persona, tal y como explica Schwartzmann (2003) (Figura 1).

Figura 1 - Calidad de vida relacionada con la salud

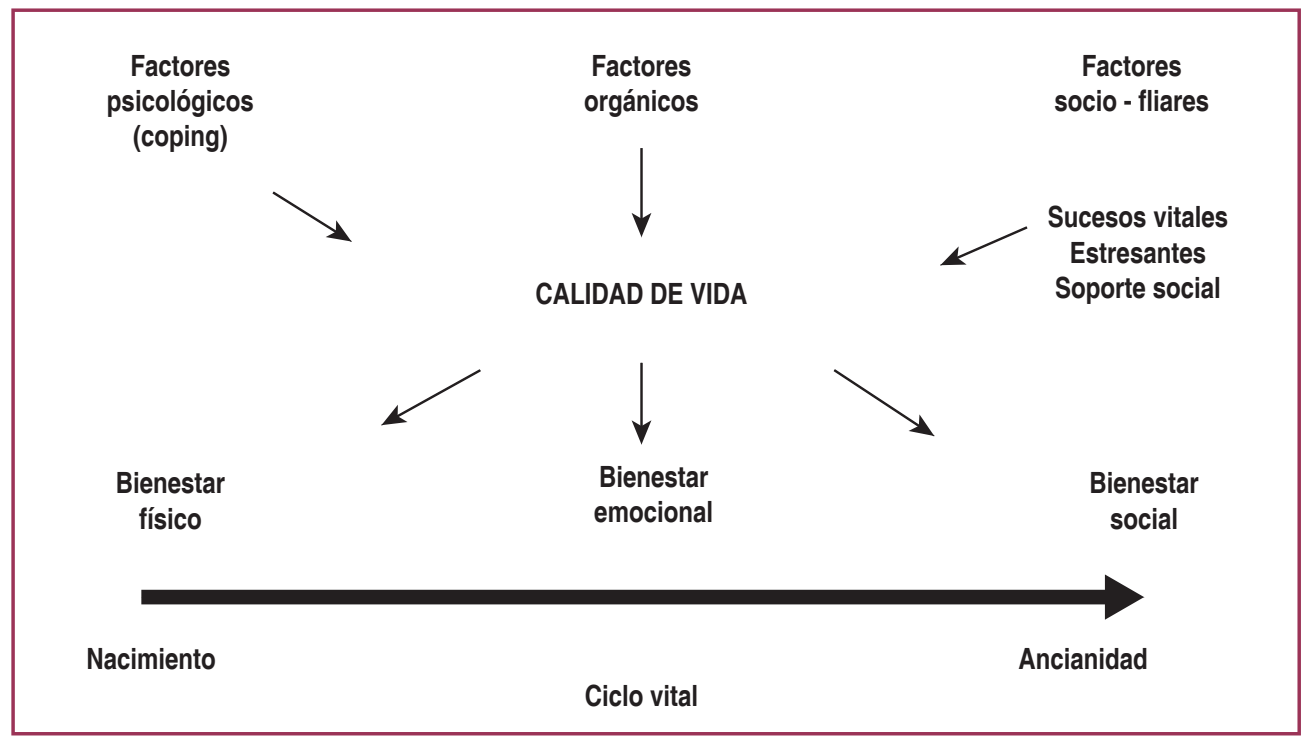


Por otra parte, tal y como exponen Castejón y Abellán (2009) la salud es por naturaleza un indicador subjetivo, aunque puede ser utilizado como predictor de futuras situaciones, de necesidades y además, avisa ya del declive en las habilidades funcionales de los ciudadanos.

En esta línea, Fernández-Mayoralas et al. (2007) y Schwartzmann (2003) señalan la importancia de incorporar a las investigaciones científicas la percepción subjetiva de las personas para predecir futuras situaciones y necesidades, así como para servir de base para diseñar y evaluar programas de intervención, debido a que es una medida válida y confiable que aporta evidencia empírica con base científica al proceso de toma de decisiones en salud (SCHWARTZMANN, 2003).

En relación al tema que nos ocupa y en la línea de lo expuesto anteriormente, se debe señalar que la salud percibida no es simplemente la ausencia de enfermedad física, pero está fuertemente influenciada por el grado de deterioro funcional y determinados factores de personalidad (BURKE et al., 2012). Estos mismos autores determinan que el estado de salud percibido declina con la edad y se asocia con mayor morbilidad, mortalidad, mala salud mental, peores resultados funcionales y mayor utilización de la atención sanitaria.

Por su parte, en la investigación desarrollada por Ruthig et al. (2008) se obtiene que mejor percepción de la salud física disminuye el riesgo estimado sobre ésta. En dicha investigación, trabajando con un rango de 2 a 5 (2 salud muy mala y 5 muy buena) estos autores obtienen una media de percepción de salud por parte de las personas mayores del $3,59 \pm 0,68$. Asimismo, en la investigación presentada por Fernández-Mayoralas et al. (2007) se obtuvieron resultados muy similares $(3,7 \pm 0,8 ; 1$ salud muy mala y 5 muy buena).

Por otro lado, el Equipo Portal Mayores (2009) obtiene que el 39,7\% de las personas mayores percibe su salud como buena o muy buena, encontrándose que las percepciones negativas aumentan con la edad y que el sexo es un factor diferenciador de la salud subjetiva, ya que el $48,5 \%$ de los varones percibe bien o muy bien su estado de salud, y sólo el $33,1 \%$ de las mujeres lo percibe de esta forma. En esta línea, Semerjian y Stephens (2007) encuentran relación estadísticamente significativa entre la edad y la percepción de su estado de salud, siendo mejor ésta en los individuos de menor edad.

Por su parte, Azpiazu et al. (2002) llevaron a cabo una investigación en la Comunidad de Madrid, en la cual obtienen que el 52,1\% de las personas mayores de 65 años perciben su salud como buena o muy buena, mientras que el $47,9 \%$ la perciben como regular, mala o muy mala, siendo estos porcentajes mayores en hombres que en mujeres.

Según Denk (2003) el concepto del objetivo imaginativo y deseable de una tercera edad activa, sana y satisfecha consigo misma, ha de integrar la actividad física y el deporte como tratamientos indispensables para las personas mayores, tanto en el área de la prevención y compensación sanitaria como en la del aumento de la mejora del bienestar general y la mejora de la independencia y la competencia de las actividades diarias.

Esta reflexión se corroboran en las investigaciones realizadas por Denk y Pache (2003) y Peerson y While (2011), en las que se concluye que aquellas personas que practican actividad física, perciben que tienen mejor estado de salud. Además encuentran una relación altamente significativa al respecto, aunque señalan que el nivel de práctica disminuye a medida que aumenta la edad. 
En este sentido, existen multitud de estudios que muestran los beneficios que la actividad física y el deporte pueden aportar a las personas mayores, no sólo en el plano físico, sino también en los planos psicológico, afectivo y social (BLAIN et al., 2000, BLAIR; BRODNEY, 1999, HOLT et al., 2001, LAURIN et al., 2001, LEMMENS et al., 2008, MALBUT-SHENNAN; YOUNG, 1991, NELSON et al., 2007, PAN et al., 1997, RADAK et al., 2007, SALVADOR et al., 1995, SANTIN-MEDEIROS; GARATACHEA, 2010, STRAWBRIDGE et al., 2002, THUNE; FURBERG, 2001, TISSANDIER et al., 2001).

No obstante, el índice de inactividad física en las personas mayores es muy elevado (AOYAGI et al., 2009, CENTRO DE INVESTIGACIONES SOCIOLÓGICAS, 2010, NELSON et al., 2007, TROIANO et al., 2008). Así, Peerson y While (2011) obtienen que en Londres, únicamente el 20,8\% de la personas mayores cumple con las recomendaciones básicas de actividad física (2,5 horas semanales o más). Cabe destacar que estos autores consideran la actividad física de caminar en su investigación.

Finalmente, se debe tener en cuenta que el principal temor ante el envejecimiento es la pérdida de salud, aspecto respondido por un $49 \%$ de las personas mayores en la encuesta publicada por el IMSERSO (ESPAÑA, 2010).

Sin embargo, no ha sido desarrollado aun ningún estudio representativo del conjunto de las personas mayores en España que permita conocer la percepción de su salud según los tipos de demanda de actividad física y deporte, ni que relacione todo ello con las diferentes variables sociodemográficas.

Por ello, y en base a la revisión de estudios previos, en la presente investigación se plantearon los siguientes objetivos:

- Conocer la salud percibida por el conjunto de las personas mayores en España, en función de los tipos de demanda de actividad física y deporte definidas por Jiménez-Beatty (2002) y Martínez del Castillo et al. (2010).

- Identificar la salud percibida por las personas mayores que practican una o más actividades físicas o deportes (demanda establecida).

- Identificar la salud percibida por las personas mayores que no practican actividad física o deporte debido a algún obstáculo o barrera, pero que están interesadas en practicar (demanda latente).

- Identificar la salud percibida por las personas mayores que no practican y que no están interesadas en realizar ninguna actividad física o deportiva (demanda ausente).

\section{MÉTODO}

El enfoque del estudio ha sido cuantitativo de corte descriptivo, transversal y el método escogido el de encuesta. En dicha encuesta, fue aplicado un cuestionario mediante entrevistas estructuradas cara a cara a una muestra aleatoria real de 933 personas de 65 años cumplidos o más residentes en España.

El universo de estudio fueron todas las personas de 65 años cumplidos 0 más empadronadas en España. Según el Instituto Nacional de Estadística español este universo es de 7.484 .392 personas, siendo mujeres el $57,72 \%$ y varones el $42,28 \%$. 


\subsection{Muestra y tipo de muestreo}

El tipo de muestreo fue probabilístico de tipo polietápico (MIQUEL et al., 2000, RODRÍGUEZ-OSUNA, 1991, SANTESMASES, 2005). En este tipo de muestreo la selección de unidades se realiza por etapas sucesivas (RODRÍGUEZ-OSUNA, 1991). En la etapa I se seleccionaron ocho municipios de forma aleatoria, concretamente se escogieron dos municipios por cada uno de los siguientes tamaños demográficos: menos de 10.000 habitantes; 10.001 a 50.000; 50.001 a 100.000; más de 100.001. En la etapa II se eligieron por sistema aleatorio simple sin reemplazamiento los barrios existentes en cada uno de los municipios en los cuales se iban a aplicar los cuestionarios. En cada uno de los barrios seleccionados, las unidades de la etapa III fueron las calles de inicio de la ruta (conjunto de diez entrevistas a aplicar en esa zona), dicha elección se efectuó al azar sobre el callejero de la ciudad. En las etapas IV, V y VI cada entrevistador/a seleccionaron el portal, la planta y la puerta de la vivienda, respectivamente, aplicando criterios de ruta aleatoria proporcionados (MIQUEL et al., 2000). Finalmente, las unidades de última etapa fueron las personas mayores entrevistadas.

El tamaño de la muestra, dado que era una población infinita o muy numerosa, y trabajando con un intervalo de confianza del 95,5\%, un margen de error permitido de muestreo del 3,27\% y suponiendo en la varianza poblacional el caso más desfavorable de $p$ igual a 50 , luego $q=50$, debía ser de 933 personas mayores (SIERRA-BRAVO, 2001).

Respecto a la afijación de la muestra, de las diferentes alternativas posibles (CEA, 2004, SIERRA-BRAVO, 2001, RODRÍGUEZ-OSUNA, 1991) se optó por una afijación proporcional a la distribución según el género de la población objeto de estudio y una afijación simple por cada tamaño demográfico. En referencia a las edades de la muestra, el $54,7 \%$ de las personas mayores tenían entre 65 y 74 años y el 45,3\% superaban los 75 años.

\subsection{Instrumento}

El Instrumento de recogida de la información utilizado fue el "Cuestionario estandarizado de Actividad Física y Personas Mayores" elaborado y validado por Graupera, Martínez del Castillo y Martín-Novo (2003). Dicho cuestionario ha sido validado y reforzado por diferentes estudios (JIMÉNEZ-BEATTY, 2002, JIMÉNEZ-BEATTY et al., 2002; JIMÉNEZ-BEATTY et al., 2007, MARTÍNEZ DEL CASTILLO et al., 2010).

Para la presente investigación se han utilizado las siguientes variables de dicho cuestionario:

- Salud percibida.

- Tipo de demanda de actividad física y deporte. Esta variable nominal tiene las tres categorías definidas anteriormente: Demanda Establecida, Demanda Latente, Demanda Ausente. Para clasificar a los sujetos, se utilizaron las respuestas a dos preguntas dicotómicas referentes a sus hábitos de actividad física semanal y a su interés en realizar actividad física semanal. En primer lugar se preguntaba: "Sin incluir el pasear ¿realiza usted alguna actividad deportiva o juegos o ejercicios físicos durante la semana?" Respuesta Sí o No (aquellas personas que contestaban sí, eran clasificadas como Demanda Establecida). A continuación, a aquellos sujetos que contestaban negativamente se les formulaba la siguiente 
pregunta: "¿Pero le gustaría realizar durante la semana alguna actividad deportiva o juegos o ejercicios físicos, sin incluir el pasear?" Respuesta Sí o No (aquellas personas que contestaban Sí, eran clasificadas como Demanda Latente; aquellas personas que contestaban No, eran clasificadas como Demanda Ausente), (MARTÍNEZ DEL CASTILLO et al., 2011).

- Variables sociodemográficas (edad, género, clase social y tamaño demográfico).

\subsection{Procedimiento}

En el trabajo de campo realizado los entrevistadores aplicaron el cuestionario estandarizado mediante entrevista personal estructurada cara a cara en el domicilio habitual de la persona mayor seleccionada. Las entrevistas tuvieron una duración promedio de 20 minutos, que oscilaban entre los 15 minutos cuando los entrevistados eran no practicantes no interesados y en torno a 25 minutos cuando los entrevistados eran practicantes y además querían realizar alguna otra actividad física, que en ese momento no podían realizar.

Durante el transcurso del trabajo de campo y a su término, se efectuaron las tareas de control y supervisión. Comprobando que las rutas y su respectivas cuotas de varones y mujeres habían sido completadas. Además, se comprobó que los cuestionarios se hallaran total y adecuadamente cumplimentados, es decir que se hubieran recogido todas las informaciones previstas y además verificando que los datos realmente hubieran sido recogidos como se debían recoger (CEA, 2004; MIQUEL et al., 2000). En los casos que no fue así, 123, se rechazaron los cuestionarios. Es por ello que la muestra real final estuvo compuesta por las ya citadas 933 personas mayores.

El artículo ha sido elaborado con los datos del Proyecto DEP2005-00161-C03-01 presentado por la Universidad Politécnica de Madrid y fue autorizado y financiado por la Dirección General de Investigación del Ministerio de Educación y Ciencia de España. En la solicitud el investigador principal debió contestar un protocolo en el que se exponía que no había ninguna implicación ética o de bioseguridad en el proyecto propuesto, teniendo en cuenta el protocolo del Comité de Ética y Bioseguridad de la Universidad Politécnica de Madrid, el proyecto fue autorizado por el Vicerrector de Investigación de dicha Universidad. Además el investigador principal del Proyecto, debió firmar en la solicitud una declaración en la que se comprometía a respetar toda la legislación vigente en materia de derechos humanos, y de Ética y Bioseguridad.

\subsection{Análisis estadístico}

Siguiendo las indicaciones para el análisis de los datos de encuesta de Cea (2004) y López-Pintor y Wert (2000) se decidió realizar un análisis descriptivo univariable y bivariable, y un análisis inferencial a través de tablas de contingencia, incluyendo el coeficiente de correlación Phi, y el valor de Chi-cuadrado de Pearson y su significación. Todo ello fue realizado mediante el paquete informático SPSS para WINDOWS (V 15.0).

\section{RESULTADOS}

Para contextualizar el presente apartado y facilitar la comprensión del resto del análisis de los resultados se debe tener en cuenta que tras analizar el tipo de demanda de actividad 
física por parte de las personas mayores en España se ha obtenido que la mayoría de las personas mayores se encuentran inmersas en el grupo referido a la demanda ausente $(69,9 \%)$, el $17,4 \%$ pertenecen a la demanda establecida y el $12,8 \%$ a la demanda latente. Cabe destacar que para el presente estudio no se ha considerado actividad física o deporte el hecho de caminar, siguiendo los estudios de García-Ferrando (2006) ya que se pretendía indagar en la práctica de ejercicio físico o deporte al margen de la actividad física realizada.

Tal y como se puede observar en la tabla 1, las personas de la demanda establecida son las que afirman en mayor medida sentirse bastante o muy satisfechas con su estado de salud, seguidas por las personas que componen el grupo de la demanda ausente, aunque con porcentajes similares a las personas integrantes de la demanda latente.

Tabla 1 - Satisfacción con estado de salud en función del tipo de demanda.

\begin{tabular}{|c|c|c|c|}
\hline \multicolumn{4}{|c|}{ Tipo de demanda } \\
\hline Satisfacción Salud (\%) & Ausente & Latente & Establecida \\
\hline Mucho & 19,2 & 14,3 & 27 \\
\hline Bastante & 43,5 & 42,9 & 52 \\
\hline Algo & 24,5 & 30,3 & 16,4 \\
\hline Nada & 12,8 & 12,6 & 3,8 \\
\hline
\end{tabular}

En primer lugar, abordando las diferentes relaciones establecidas con los tipos de demanda y las variables sociodemográficas, se van a analizar los resultados obtenidos en torno al preocupante $69,9 \%$ de personas mayores que conforman la demanda ausente (tabla 2). Se puede apreciar que los hombres, las personas que se consideran de clase alta y las de menor edad son las que están más satisfechas con su estado de salud. En cuanto a los diferentes estratos demográficos, se puede observar que son las personas de los municipios grandes las que mayor porcentaje presentan al afirmar estar algo o nada satisfechas con su estado de salud (48.6\% en total). Asimismo, se han obtenido relaciones estadísticamente significativas en todos los casos excepto en la relación con la variable edad.

Tabla 2 - Salud percibida por las personas mayores en la demanda ausente en función de las características sociodemográficas.

\begin{tabular}{|c|c|c|c|c|c|c|c|c|c|c|c|}
\hline \multicolumn{12}{|c|}{ Demanda Ausente } \\
\hline \multirow{2}{*}{$\begin{array}{l}\text { Satisfacción } \\
\text { Salud (\%) }\end{array}$} & \multicolumn{2}{|c|}{ Género } & \multicolumn{3}{|c|}{ Clase social } & \multicolumn{2}{|c|}{ Edad } & \multicolumn{4}{|c|}{ Tamaño demográfico } \\
\hline & $\mathrm{H}$ & M & Baja & Media & Alta & $<74$ & $>75$ & $<10.000$ & $\begin{array}{l}10.000- \\
50.000\end{array}$ & $\begin{array}{l}50.000- \\
100.000\end{array}$ & $>100.000$ \\
\hline Mucho & 23,2 & 15,4 & 18,8 & 16,5 & 42,6 & 20,6 & 17,9 & 19 & 21,3 & 25,3 & 10,3 \\
\hline Bastante & 46,3 & 40,8 & 39,8 & 46,9 & 38,3 & 45 & 42,1 & 45,3 & 37,4 & 49,4 & 41,1 \\
\hline Algo & 20,3 & 28,4 & 25,2 & 25,7 & 12,8 & 24,8 & 24,2 & 24,6 & 24,5 & 15,7 & 34,2 \\
\hline Nada & 10,2 & 15,4 & 16,5 & 10,9 & 6,4 & 9,6 & 15,8 & 11,2 & 16,8 & 9,6 & 14,4 \\
\hline Significación & \multicolumn{2}{|c|}{$\begin{array}{c}{ }^{*} \Phi=, 15 ; \\
p=,<01\end{array}$} & \multicolumn{3}{|c|}{${ }^{*} \Phi=, 20 ; p=,<01$} & \multicolumn{2}{|c|}{$\begin{array}{c}\Phi=, 09 \\
p=, 13\end{array}$} & \multicolumn{4}{|c|}{${ }^{*} \Phi=, 21 ; p=,<01$} \\
\hline
\end{tabular}


En cuanto a las personas de la demanda latente, en la tabla 3 se puede observar que nuevamente los hombres están bastante o muy satisfechos con su estado de salud en mayor medida que las mujeres (61,4\% y $53,2 \%$ respectivamente) y que a medida que aumenta la clase social percibida, se obtienen mayores porcentajes en cuanto a personas muy o bastante satisfechas con su estado de salud (51,4\% en las personas de clase social baja; $57,9 \%$ en las de clase media y $61,6 \%$ en las de clase alta). Respecto a la edad, se aprecian menores diferencias que en la demanda ausente, ya que aunque las personas de menor edad son las que afirman en mayor medida estar muy satisfechas con su estado de salud, las personas de mayor edad presentan porcentajes muy superiores al afirmar estar bastante satisfechas con éste. Finalmente, al relacionar la variable objeto de estudio con el tamaño demográfico, se puede apreciar que es en el estrato de los municipios pequeños en el que las personas mayores afirman en mayor medida estar muy o bastante satisfechas con su estado de salud. Cabe destacar que únicamente se ha obtenido relación estadísticamente significativa al relacionar esta última variable con la salud percibida, aspecto que puede ser explicado por el bajo porcentaje de personas del grupo de la demanda latente.

Tabla 3 - Salud percibida por las personas mayores en la demanda latente en función de las características sociodemográficas.

\begin{tabular}{|c|c|c|c|c|c|c|c|c|c|c|c|}
\hline \multicolumn{12}{|c|}{ Demanda Latente } \\
\hline \multirow{2}{*}{$\begin{array}{l}\text { Satisfacción } \\
\text { Salud (\%) }\end{array}$} & \multicolumn{2}{|c|}{ Género } & \multicolumn{3}{|c|}{ Clase social } & \multicolumn{2}{|c|}{ Edad } & \multicolumn{4}{|c|}{ Tamaño demográfico } \\
\hline & $\mathrm{H}$ & M & Baja & Media & Alta & $<74$ & $>75$ & $<10.000$ & $\begin{array}{l}10.000- \\
50.000\end{array}$ & $\begin{array}{l}50.000- \\
100.000\end{array}$ & $>100.000$ \\
\hline Mucho & 14 & 14,5 & 14,3 & 13 & 23,1 & 16,7 & 9,8 & 18,2 & 32,3 & 16 & 1,9 \\
\hline Bastante & 47,4 & 38,7 & 37,1 & 44,9 & 38,5 & 38,5 & 51,2 & 63,6 & 35,5 & 40 & 44,2 \\
\hline Algo & 28,1 & 32,3 & 28,6 & 31,9 & 30,8 & 30,8 & 29,3 & 18,2 & 16,1 & 28 & 42,3 \\
\hline Nada & 10,5 & 14,5 & 20 & 10,1 & 7,7 & 14,1 & 9,8 & 0 & 16,1 & 16 & 11,5 \\
\hline Significación & \multicolumn{2}{|c|}{$\Phi=, 09 ; p=, 78$} & \multicolumn{3}{|c|}{$\Phi=, 77 ; p=, 17$} & \multicolumn{2}{|c|}{$\Phi=, 14 ; p=, 50$} & \multicolumn{4}{|c|}{${ }^{\star \star} \Phi=, 42 ; p=, 01$} \\
\hline
\end{tabular}

Atendiendo a la salud percibida por las personas mayores del grupo de la demanda establecida (tabla 4), se puede apreciar que en general las mujeres están más satisfechas con su estado de salud que los hombres, a diferencia de lo que ocurre en los otros dos grupos de demanda. En relación a la clase social, son las personas de clase alta las que mayor porcentaje presentan al afirmar sentirse muy satisfechas con su estado de salud, aunque las personas de clase media y baja son las que en mayor medida afirman sentirse bastante satisfechas con el mismo. En este sentido se aprecian menores diferencias entre clases que en el resto de grupos. Además, cabe destacar que las personas de ambos grupos de edad de la demanda establecida presentan porcentajes similares al ser cuestionados por la satisfacción con su estado de salud actual, y que las personas de los municipios más pequeños son las que en menor medida afirman estar algo o nada satisfechos con su estado de salud. Al igual que en la demanda latente, únicamente se ha obtenido relación estadísticamente significativa al relacionar esta última variable con la salud percibida, aspecto que nuevamente podría ser explicado por el bajo porcentaje de personas practicantes de actividad física o deporte en España. 
Tabla 4 - Salud percibida por las personas mayores en la demanda establecida en función de las características sociodemográficas.

\begin{tabular}{|c|c|c|c|c|c|c|c|c|c|c|c|}
\hline \multicolumn{12}{|c|}{ Demanda Establecida } \\
\hline \multirow{2}{*}{$\begin{array}{l}\text { Satisfacción } \\
\text { Salud (\%) }\end{array}$} & \multicolumn{2}{|c|}{ Género } & \multicolumn{3}{|c|}{ Clase social } & \multicolumn{2}{|c|}{ Edad } & \multicolumn{4}{|c|}{ Tamaño demográfico } \\
\hline & $\mathrm{H}$ & M & Baja & Media & Alta & $<74$ & $>75$ & $<10.000$ & $\begin{array}{l}10.000- \\
50.000\end{array}$ & $\begin{array}{l}50.000- \\
100.000\end{array}$ & $>100.000$ \\
\hline Mucho & 25,4 & 28,1 & 23,5 & 27,3 & 31,6 & 28,4 & 23,3 & 28,6 & 51,2 & 26,4 & 5,9 \\
\hline Bastante & 49,2 & 55,2 & 50 & 55,6 & 36,8 & 51,7 & 55,8 & 66,7 & 41,2 & 54,7 & 58,8 \\
\hline Algo & 22,2 & 12,5 & 17,6 & 15,2 & 26,3 & 16,4 & 16,3 & 4,8 & 11,8 & 13,2 & 35,3 \\
\hline Nada & 3,2 & 4,2 & 8,8 & 2 & 5,3 & 3,4 & 4,7 & 0 & 5,9 & 5,7 & 0 \\
\hline Significación & \multicolumn{2}{|c|}{$\begin{array}{c}\Phi=, 13 \\
p=, 45\end{array}$} & \multicolumn{3}{|c|}{$\Phi=, 19 ; p=, 46$} & $\begin{array}{l}\Phi= \\
\mathrm{p}=\end{array}$ & $\begin{array}{l}06 ; \\
91\end{array}$ & \multicolumn{4}{|c|}{${ }^{* *} \Phi=, 39 ; p=,<01$} \\
\hline
\end{tabular}

Relación estadísticamente significativa: *baja o ligera; ** moderada.

Fuente: Elaboración propia

\section{DISCUSIÓN}

En relación a la práctica físico-deportiva de las personas mayores, los resultados obtenidos en la demanda establecida corroboran el gran índice de inactividad encontrado en la literatura científica (AOYAGI et al., 2009, CENTRO DE INVESTIGACIONES SOCIOLÓGICAS, 2010; NELSON et al., 2007; TROIANO et al., 2008).

Atendiendo a la percepción del estado de salud en función del tipo de demanda, se ha obtenido que las personas practicantes de ejercicio físico o deporte son las que en mayor porcentaje afirman estar bastante o muy satisfechas con su estado de salud, coincidiendo con los resultados obtenidos en otras investigaciones (COCHRANE et al., 1998; DENK y PACHE, 2003; PEERSON y WHILE, 2011; SEGUIN et al., 2010). Cabe destacar que los resultados obtenidos entre las personas de las demandas ausente y latente son muy similares, lo que podría explicar que ante una percepción no muy positiva del estado de salud algunas personas mayores se plantean realizar actividad física, aunque no pueden hacerlo por alguna barrera y otras señalan que no desean ser practicantes de actividad física, siendo éste uno de los motivos encontrados en otras investigaciones (MARTíNEZ DEL CASTILLO, 2003).

En relación a las variables sociodemográficas género y edad, en la presente investigación se ha obtenido que los hombres y las personas de menor edad son las menos satisfechas con su estado de salud en el grupo de la demanda ausente y en el de la demanda latente, aunque si se analizan los resultados se pueden observar menores diferencias en este último grupo de demanda. Igualmente en las investigaciones de Azpiazu et al. (2002), Equipo Portal Mayores (2009), Fernández-Mayoralas et al. (2007), I.N.E. (2011-2012) y Semerjian y Stephens (2007) se obtiene que la valoración del estado de salud es significativamente diferente por género y por tramos de edad, ya que las mujeres valoran su salud peor que los hombres y los más mayores valoran peor su salud que los más jóvenes.

No obstante, en el grupo de la demanda establecida se invierten estos resultados, es decir, entre las personas practicantes de ejercicio físico o deporte se obtiene que las mujeres están más satisfechas con su estado de salud y que las personas del grupo de menor edad presentan porcentajes muy similares a los mayores, afirmando además en mayor medida estar muy satisfechos con su estado de salud. Igualmente, en el estudio de Michalos et al. (2007) las mujeres son ligeramente más activas físicamente que los hombres. 
Estos Resultados coinciden con los obtenidos por Denk y Pache (2003), aspecto por el cual estos autores señalan que los deportistas activos tienen un autoconcepto más positivo en relación a su estado de salud y que la actividad físico-deportiva regular mejora objetivamente la salud. No obstante, cabe señalar que los resultados obtenidos en la Encuesta Nacional de Salud (I.N.E., 2011-2012) muestran que tanto las mujeres como las personas de más edad son las más sedentarias.

Respecto a la variable sociodemográfica clase social, en la presente investigación se ha obtenido que a medida que aumenta la clase social percibida, aumenta el porcentaje de personas que afirman estar muy o bastante satisfechas con su estado de salud en la demanda latente $(51,4 \%$ clase social baja; $57,9 \%$ media y $61,6 \%$ alta) y en la demanda ausente $(58,6 \%$ baja; $63,4 \%$ media y $80,9 \%$ alta). Estos datos coinciden con los obtenidos en la Encuesta Nacional de Salud (I.N.E., 2011-2012), donde también se obtiene una mayor percepción del estado de salud a medida que se aumenta la clase social basada en el puesto de trabajo desempeñado. Cabe destacar que en dicha investigación también se observa una tendencia claramente creciente al sedentarismo a medida que disminuye la clase social.

No obstante, en el grupo de la demanda establecida son las personas que se consideran de clase media las que mejor perciben su estado de salud, seguidas de las personas de clase baja. Por su parte, Fernández-Mayoralas et al. (2007) detectan una tendencia creciente de las valoraciones positivas conforme se avanza desde la clase social más baja (56\%) hasta la media/alta (77\%), aunque en la clase alta se interrumpe con un descenso en el porcentaje de mayores que describen su salud como buena y muy buena (68\%).

En este sentido, en la investigación de Martínez del Castillo et al. (2010) se obtuvo que a medida que disminuye la clase social percibida disminuye también el porcentaje de personas practicantes de actividad física y deporte.

Finalmente, se ha obtenido una relación estadísticamente significativa al relacionar el tamaño del municipio de residencia con la satisfacción con el estado de salud de las personas mayores en todos los grupos de demanda. Así, se puede apreciar que en los grupos de la demanda ausente y establecida a medida que aumenta el tamaño del municipio disminuye el número de personas que afirman estar bastante o muy satisfechas con su estado de salud. En cambio, en el grupo de la demanda ausente las personas más satisfechas con éste son las de los municipios de entre 50.000 y 100.000 , seguidas de las de los municipios pequeños. Estos resultados son similares a los obtenidos en la Encuesta Europea de Salud Pública en España (I.N.E., 2009) encontrándose que la mayor percepción del estado de salud como muy buena se encuentra en los municipios medianos, aunque los resultados son muy similares en todos los estratos.

En conclusión, se puede afirmar que las personas mayores practicantes de actividad física o deporte están más satisfechas con su estado de salud que las no practicantes y que en este grupo de personas las posibles diferencias entre las variables sociodemográficas en cuanto a la satisfacción con el estado de salud dejan de existir, tendiendo a invertirse en algunos casos.

Finalmente debe tenerse en cuenta que la gran mayoría de las personas mayores en España se encuentran inmersas en el grupo de demanda ausente. Por ello, sería interesante realizar una futura investigación teniendo en cuenta únicamente a personas que desean practicar actividad física y deporte, pero que por algún obstáculo o barrera no pueden 
participar (demanda latente) y a personas practicantes de actividad físico-deportiva (demanda establecida) con el objetivo de aumentar la muestra de ambos grupos.

\section{REFERÊNCIAS}

AOYAGI, Yukitoshi et al. Habitual Physical activity and physical fitness in older japanese adults: the Nakanojo Study. Gerontology, Basel, v. 55, p. 523-531, 2009.

AZPIAZU, Mercedes et al. Factores asociados a mal estado de salud percibido o a mala calidad de vida en personas mayores de 65 años. Revista Española de Salud Pública, Madrid, v. 76, n. 6, p. 683-699, 2002.

BLAIN, Hugues et al. Les effets preventifs de l'activite physique chez les personnes agees. Presse Médicale, Paris, v. 29, p. 1240-1248, 2000.

BLAIR, Steven; BRODNEY, Suzzane. Effects of physical inactivity and obesity on morbidity and mortality: current evidence and research issues. Medicine and Science in Sports Exercise, Indianapolis, v. 31, n. 11, p. S646-S662, 1999.

BURKE, Kate E. et al.Factors associated with perceived health in older adult Irish population. Aging \& Mental Health, London, v. 6, n. 3, p. 288-295, 2012.

CAMPOS, Antonio. et al. Demanda y percepción del monitor de las personas mayores en la actividad física y deporte en España. Revista de Psicología del Deporte, Palma, v. 20, n. 1, p. 61-77, 2011.

CASTEJÓN, Penélope.; ABELLÁN, Antonio. Estado de salud. In: DÍAZ, Rosa (Coord.). Las personas mayores en España: datos estadísticos estatales y por Comunidades Autónomas. Informe 2008. Madrid: Ministerio de Sanidad y Política Social, 2009. p. 67-132.

CEA, María A. Métodos de encuesta. Madrid: Síntesis, 2004.

CENTRO DE INVESTIGACIONES SOCIOLÓGICAS. Hábitos deportivos en España. 2010. Disponible en: <http://www.cis.es/cis/opencms/-Archivos/Marginales/2820 2839/2833/Es2833.pdf>. Acceso en: 10 sept. 2014.

COCHRANE, Thomas et al. Exercise, Physical Function and Health Perceptions of Older People. Physiotherapy, London, v. 84, n. 12, p. 598-602, 1998.

DENK, Heinz. Introducción a la edición en alemán. In: DENK, Heinz (Coord.). Deporte para mayores. Barcelona: Paidotribo, 2003. p. 5-7.

DENK, Heinz; PACHE, Dieter. Actitud de las personas mayores frente al ejercicio y a la actividad deportiva. In: DENK, Heinz (Coord.). Deporte para mayores. Barcelona: Paidotribo, 2003. p. 43-67.

EQUIPO PORTAL MAYORES. Un perfil de las personas mayores en España, 2009: Indicadores estadísticos básicos. Informes Portal Mayores, p. 97, 2009. Disponible en: <http://www. imsersomayores.csic.es/documentos/documentos/pmindicadoresbasicos09.pdf.> Acceso en: 10 sept. 2014. 
FERNÁNDEZ-MAYORALAS, Gloria, et al. El significado de la salud en la calidad de vida de los mayores. Informes Portal Mayores, p. 74, 2007. Disponible en: <http://www.imsersomayores. csic.es/documentacion/estudiosyresultados/informes/index.html>. Acceso en: 10 sept. 2014.

GARCÍA, Francisco. Introducción: vejez, envejecimiento e historia. La edad como objeto de investigación. In: F. GARCÍA, Francisco (Coord.). Vejez, envejecimiento y sociedad en España: siglos XVI-XXI. Cuenca: Universidad de Castilla-La Mancha, 2005. p. 11-34.

GARCÍA-FERRANDO, Manuel. Posmodernidad y deporte: entre la individualiuzación y la masificación. encuesta hábitos deportivos de los españoles 2005, Madrid: Siglo XXI, 2006.

GRAUPERA, José L.; MARTÍNEZ DEL CASTILLO, Jesús; MARTÍN-NOVO, Beatriz. Factores motivacionales, actitudes y hábitos de práctica de actividad física en las mujeres mayores. Serie ICD de Investigación en Ciencias del Deporte, Madrid, v. 36, p. 181-222, 2003.

HOLT, Richard, I.G.; WEBB, Emma; PENTECOST, Claire; SÖNKSEN, Peter, H. Aging and physical fitness are more important than obesity in determining exercise-induced generation of GH. The Journal of Clinical Endocrinology and Metabolism, Baltimore, v. 86, n. 12, p. 5715-5720, 2001.

ESPAÑA. Ministerio de Sanidad y Politca Social. Encuesta mayores 2010. Madrid: Ministerio de sanidad y política social, 2010. Disponible en: <http://www.imserso.es/InterPresent2/groups/ imserso/documents/binario/presentacionencuestamayores 20.pdf>. Acceso en: 10 sept. 2014.

INSTITUTO NACIONAL DE ESTADÍSTICA. Encuesta europea de salud en España 2009. Madrid: INE, 2009. Disponible en: <http://www.ine.es/jaxi/menu.do?type=pcaxis\&path=\%2Ft15/ p420\&file=inebase >. Acceso en: 10 sept. 2014.

INSTITUTO NACIONAL DE ESTADÍSTICA. Encuesta nacional de salud: año 2011-2012. Madrid: INE, 2012 Disponible en: <http://www.ine.es/jaxi/menu.do?type=pcaxis\&path=/t15/ p419\&file=inebase $>$. Acceso en: 10 sept. 2014.

JIMÉNEZ-BEATTY, José E. La demanda de servicios de actividad física en las personas mayores. Tesis (doctoral). León, 2002. Universidad de León, León, 2002.

JIMÉNEZ-BEATTY, José E. et al. Motivational factors and physician's advice in physical activity in the older urban population. Journal of Aging and Physical Activity, Champaign, v. 15, n. 3 , p. 241-256, 2007.

JIMÉNEZ-BEATTY, José E. et al. La dirección de programas de actividad física ante las barreras para la incorporación de la tercera edad. In: CONGRESO INTERNACIONAL DE ACTIVIDAD FÍSICA Y DEPORTIVA PARA PERSONAS MAYORES, 1., 2002, Málaga.

LAURIN, Danielle; VERREAULT, René; LINDSAY, Joan; MACPHERSON, Kathleen; ROCKWOOD, Kenneth. Physical activity and risk of congnitive impairment and dementia in elderly persons. Archives of neurology, Chicago, v. 58, p. 498-504, 2001.

LEMMENS, Vepp et al. A systematic review of the evidence regarding efficacy of obesity prevention interventions among adults. Obesity Reviews, Oxford, v. 9, p. 446-455, 2008.

LÓPEZ PINTOR, Rafael; WERT, José I. El análisis de datos de encuesta. In: GARCÍA, Manuel; IBÁÑEZ, Jesús; ALVIRA, Francisco (Ed.). El análisis de la realidad social: métodos y técnicas de investigación. Madrid: Alianza, 2000. p. 525-554. 
MALBUT-SHENNAN, K.; YOUNG, A. The physiology of physical performance and training in old age. Coronary Artery Disease, Vermont, v. 10, n. 1, p. 41-42. 1991.

MARTÍNEZ DEL CASTILLO, Jesús. El deporte para todos en la tercera edad. In: CONGRESO Y ASAMBLEA DE DEPORTE PARA TODOS, 2., 2002, Madrid. p. 173-190.

MARTÍNEZ DEL CASTILLO, Jesús et al. Being physically active in old age: relationships with being active earlier in life, social status and agents of socialisation. Ageing and Society, Cambridge,v. 30, p. 1097-1113, 2010.

MARTÍNEZ DEL CASTILLO, Jesús et al. La recomendación médica y el tipo de demanda de actividad física en las personas mayores de la provincia de Guadalajara. Revista Internacional de Ciencias del Deporte, Murcia, v. 7, n, 23, p. 91-102, 2011.

MICHALOS, Alex.C. et al. Health and quality of life of older people, a replication after six years. Social Indicators Research, Dordrecht, v. 84, p. 127-158, 2007.

MIQUEL, Salvador et al. Investigación de mercados. Madrid: Mc Graw-Hill, 2000.

NELSON, Miriam E et al. Physical Activity and Public Health in Older Adults. Recommendation From the American College of Sports Medicine and the American Heart Association.

Circulation: Journal of the American Heart Association, Baltimore, v. 116, p. 1094-1105, 2007.

PAN, X. R. et al. Effects of diet and exercise in preventing IDDM in people with impaired glucose tolerance. The Da Qing IGT and Diabetes study. Diabetes Care, Alexandria, v. 20, p. 537-544, 1997.

PEERSON, Ann.; WHILE, Alison. Physical activity among older people and related factors.

Health Education Journal, London, v. 71, n. 2, p. 144-153, 2011.

RADAK, Zsolt et al. Effects of exercise on brain function: role of free radicals. Applied Physiology Nutrition Metabolism, Washington, v. 32, n. 5, p. 942-6, 2007.

RODRÍGUEZ-OSUNA, Jacinto. Métodos de muestreo. Madrid: Centro de Investigaciones Sociológicas, 1991.

ROMERO, Santiago. Ejercicio físico y salud en edad avanzada. In: 1er CONGRESO INTERNACIONAL DE LA ACTIVIDAD FÍSICA Y DEPORTIVA PARA PERSONAS MAYORES., 1., 2002, Málaga. p. 201-216.

RUTHIG, Joelle C.; CHIPPERFIELD, Judith G.; BAILIS, Daniel S.; PERRY, Raymond P. Perceived Control and Risk Characteristics as Predictors of Older Adults' Health Risk Estimates. The Journal of Social Psychology, Washington, v. 148, n. 2, p. 667-688, 2008.

SALVADOR, Alicia; SUAY, Ferran; MARTÍNEZ-SANCHÍS, Sonia; GONZÁLEZ-BONO, Esperanza; RODRÍGUEZ, Marta; GILABERT, Alfredo. Deporte y salud: efectos de la actividad deportiva sobre el bienestar psicológico y mecanismos hormonales subyacentes. Revista de Psicología General y Aplicada, Madrid, v. 48, p. 125-137. 1995.

SANTESMASES, Miguel. DYANE Versión 3 Diseño y análisis de encuestas en investigación social y de mercados. Madrid: Ediciones Pirámide. 2005.

SANTIN-MEDEIROS, Fernanda; GARATACHEA, Nuria. Efectos musculoesqueléticos del entrenamiento con vibraciones en ancianos. Revista Española de Geriatría y Gerontología, Madrid, v. 45, n. 5, p. 281-284. 2010. 
SCHWARTZMANN, Laura. Calidad de vida relacionada con la salud: aspectos conceptuales. Ciencia y Enfermería IX, Concepción, v. 2, p. 9-21, 2003.

SEGUIN, Rebeca A. et al. Strength Training and Older Women: A Cross-Sectional Study Examining Factors Related to Exercise Adherence. Journal of Aging and Physical Activity, Champaign, v. 18, n. 2, p. 201-208, 2010.

SEMERJIAN, Tamar; STEPHENS, Dawn. Comparison Style, Physical Self-Perceptions, and Fitness Among Older Women. Journal of Aging and Physical Activity, Champaign , v. 15, p. 219-235, 2007.

SIERRA-BRAVO, Restituto. Técnicas de investigación social: teoría y ejercicios. Madrid: Paraninfo, 2001.

STRAWBRIDGE, William J; DELEGER, Stéphane; ROBERTS, Robert E.; KAPLAN, George A. Physical Activity Reduces the Risk of Subsequent Depression for Older Adults. American Journal of Epidemiology, Baltimore, v. 156, n. 4, p. 328-334, 2002.

THUNE, Inger; FURBERG, Anne-Sofie. Physical activity and cancer risk: dose-response and cancer, all sites and site-specific. Medicine and Science in Sports and Exercise, Hagerstown, v. 33, n. 6, p. S530-S550, 2001.

TISSANDIER, O. et al. Testosterone, dehydroepiandrosterone, insulin-like growth factor 1, and insulin in sedentary and hysically trained aged men. European Journal of Applied Physiology, Heidelberg, n. 85, v. 1-2, p. 177-84, 2001.

TROIANO, Richard et al. Physical Activity in the United States Measured by Accelerometer. Medicine and Science in Sports and Exercise, Hagerstown,v. 40, p. 181-188. 2008. 